International Journal of Biotechnology and Biochemistry.

ISSN 0973-2691 Volume 11, Number 2 (2015) pp. 105-114

(C) Research India Publications

https://dx.doi.org/10.37622/IJBB/11.2.2015.105-114

\title{
Efficiency of Liposomal Drug Delivery System Against Plasmodium In Vitro and In Vivo Culture
}

\author{
Pallav Kaushik Deshpande ${ }^{\mathbf{1}}$, Ragini Gothalwal ${ }^{\mathbf{1}}$, Anupam Kumar Pathak ${ }^{2}$ \\ Department of Biotechnology, Barkatullah University,Bhopal,(M.P):462026 \\ Department of Pharmacy, Barkatullah University, Bhopal. (M.P):462026
}

\begin{abstract}
Liposomes are the leading drug delivery systems for the systemic (intravenous) administration of drugs. Liposome encapsulation can markedly alter the biodistribution and pharmacokinetics of well-known chemotherapeutic agents. Present work explore liposomal drug delivery system for anti-malarial drugs with the objective of protection of the drug from the degradation and the sustained release of the drug along with minimization of their adverse effects. Azadirachta indica, Cinchona officinalis and Artemisia annua having anti plasmodium activities and chloroquine, mefloquine, artesunate synthetic drugs were selected in the study. In vitro Plasmodium culture was maintained by modified method Trager and Jenson (1997). In vivo Culture were maintained and screened as method performed by Peter et. al.,(1975) . Effect of antimalarial (natural and synthetic) molecule entrapped in liposome and in free form were measured. Results indicated that synthetic as well as plant extracts exhibits better response when given in liposomal form, both in vivo and in vitro conditions. Which is in accordance to ability of liposomes to entrap hydrophilic and hydrophobic drugs with concomitant reduction in their toxicity potential, their versatility and their amenability for surface modification are the major factors responsible for their popularity in drug delivery research.
\end{abstract}

Key words: Liposome, Plasmodium,, Drug Delivery System, Azadirachta indica, Cinchona officinalis, Artemisia annua

\section{Introduction}

The concept of drug targeting, suggested by Paul Enrich, considered a hypothetical 'magic bullet' as an entity consisting of two components the first one should recognize and bind the target while the second one should provide a therapeutic action in this target. Currently, the concept of magic bullet includes a coordinated behavior of three 
components: one drug two targeting moiety and three pharmaceutical carriers. Drug targeting evolved as the most desirable but elusive goal in drug delivery science. Liposomes are, perhaps, the earliest type of nanomaterial developed for drug delivery (Maurer et al., 2001). They are vesicles composed of one (unilamellar) or several (multilamellar) lipid bilayers surrounding internal aqueous compartments. Relatively large amounts of drug molecules can be incorporated into the aqueous compartment (water soluble compounds) or lipid bilayersn (lipophilic compounds).

\section{Material and Methods}

\section{Plant material}

Plant selected for antiplasmodium activity: Artemisia annua, Cinchona officinalis, Azadirachta indica

\section{Preparation And Extraction Procedure For Plant Material}

Prepration of plant material.

Materials of the three plants selected for the present study were grinded to coarse powder and stored in airtight containers at room temperature in the dark until used.(Trease and Evans 1978).

\section{Method For Alcoholic Extraction}

\section{Extraction for Cinchona officinalis}

The powdered Cinchona bark (50g) was mixed with calcium oxide (30gms), water $(40 \mathrm{ml})$ and $5 \%$ sodium hydroxide to form a paste and kept overnight.Then obtained paste was packed in Soxhlet apparatus and extracted with methanol for $8 \mathrm{hrs}$ at $60^{\circ} \mathrm{C}$.Methanolic extract was shaken with three successive portions of $25 \mathrm{ml}$ of $5 \%$ sulphuric acid. The acidified extract was basified with ammonia solution ( $\mathrm{pH} 10)$ and liberated alkaloids were extracted with three successive $20 \mathrm{ml}$ portion of chloroform. The chloroform extracts were combined. The chloroform was distilled off to yield total alkaloids.It was dried to constant weight at $80^{\circ} \mathrm{C}$ and kept in dessicator (Kokate, 2009). Then stored at $4^{\circ} \mathrm{Cuntil}$ use in sealed bottle, protect against light and moisture.

\section{Extraction of Azadirachta Indica}

$50 \mathrm{~g}$ of air-dried powder was soaked in ethyl alcohol $(100 \mathrm{ml})$ and kept forovernight.Then it was filtered through 8 layers of muslin cloth, filtrate was collected andresidue was discarded.Filtrate was packed in Soxhlet apparatus and extraction was done for $8 \mathrm{hrs}$ at $80-90^{\circ} \mathrm{C}$. Solvent was distilled off to one -fourth to yield alcoholic extract. 


\section{Extraction for Artimisia annua}

Artemisinin, a sesquiterpene lactone is an antimalarial constituent of Artimisia annua. Extraction process which was followed in present study is: Dried leaves of artimisia annua are coarsely powdered and extracted with the help of petroleum ether (over night). Petroleum ether was then filtered then concentrated to dryness. Concentrated extract found was then dissolved in chloroform. Aerial part of Artemisia annua were dried in shade, grounded to coursed powder stored in air tight amber colored bottle. Artemisinin, a sesquiterpene lactone is an antimalarial constituent of Artimisia annua. Extraction process which was followed in this study.Dried leaves of Artimisia annua were coarsely powdered and extracted with the help of petroleum ether (over night). Petroleum ether was then filtered then concentrated to dryness with the help soxhlet then concentrated extract found was then dissolved in chloroform. Acetonitrile was added to the chloroformic solution which is responsible for the sepration of impurities and pricipetation of waxes. Filtration was done to separate out the impurities. The filtrate was again concentrated and was subjected to cool down. Crystals of artimisinin were deposited which were further purified by washing and recrystallization with alcohol. .(Trease and Evans 1978, Kokate, 2009).

\section{Method for Aqueous extraction}

The powdered material of all the selected plants $(50 \mathrm{~g})$ separately were soaked in water $(100 \mathrm{ml})$ and kept for overnight. Then it was filtered through 8 layers of muslin cloth, filtrate was collected and residue was discarded. Filtrate was packed in Soxhlet apparatus and extraction was done for $6-8 \mathrm{hrs}$ at $80-90^{\circ} \mathrm{C}$. Solvent was distilled off to one -fourth to yield aqueous extract. Collected extract was then weighed and stored at $4^{\circ} \mathrm{C}$ until use.

\section{Liposomes Preparation}

Liposomes were prepared using the thin film hydration technique. Briefly, $100 \mathrm{mg} \mathrm{ml}^{-}$ ${ }^{1}$ of phospholipids in chloroform taken in a clean moisture-free container was purged with nitrogen gas to remove the solvent. $5 \mathrm{ml}$ of phosphate buffered saline (PBS pH 7.4) were added to the container and the mixture was warmed at $60^{\circ} \mathrm{C}$ for 30 minutes. The solution was then extruded through polycarbonate membranes of $200 \mathrm{~nm}$ pore size using an extruder for ten cycles to obtain extruded liposomes(Maurer et al., 2001). The liposomes were lyophilized and stored at $-20^{\circ} \mathrm{C}$ in air-tight vials.

\section{Preparation of Host Erythrocytes}

Human erythrocytes for parasite culture are prepared by drawing blood into heparintreated tubes and washing several times in RPMI 1640 medium to separate the erythrocytes from the plasma and buffy coat. Separation can be achieved by centrifuging the blood at $3500 \mathrm{rpm}$ for 8 minutes at $4^{\circ} \mathrm{C}$ in a swing-out rotor (centrifuged machine). Leukocyte-free erythrocytes are typically stored at $50 \%$ hematocrite (i.e. 1 volume of malaria culture media for 1 volume of packed erythrocytes, corresponding to approximately $5 \times 10^{9}$ cells ml $^{-1}$. (Trager et.al., 1997) 


\section{Culture Media}

RPMI 1640 medium containing L-glutamine (High Media), 25mM HEPES (CDH), 10 $\mu \mathrm{g} \mathrm{ml}^{-1}$ gentamicin $(\mathrm{CDH}), 0.225 \% \mathrm{NaHCO} 3(\mathrm{CDH})$. Medium was adjusted to a $\mathrm{pH}$ of 7.3 to 7.4. Once media was prepared media was filtered through $0.22 \mu \mathrm{m}$ syringe filters then stored in air tight plastic bottles, at $4-8^{\circ} \mathrm{C}$ till further use. During culture maintenance media was supplemented with $5 \%$ fresh human serum $(\mathrm{O}+\mathrm{blood}$ group) (Trager et. al., 1997).

\section{Procurement of Experimental Animal}

After taking permission for animal studies from Institutional Animal Ethics Committee (IACE), B.U, Bhopal. (Reg No. CPCSEA/444), Swiss albino mice, weighing 27-35 g and age of 6-8 weeks, were selected for the study, The mice were obtained from the local breeder and maintained in animal house of Department of pharmacy, Barkatullah University, Bhopal. Animals were housed in propylene cages with standard animal house under natural $12 \mathrm{~h}$ light and 12 dark cycles at $24-28^{\circ} \mathrm{C}$ temperature. They were maintained on standard pallet diet (Hindustan lever Ltd. Bangalore) and water ad labium. They have free access to food and water. The animals were acclimatized to the test environment for one week before starting the experiment. Animals were fasted for at least 12 hours before the onset of each activity. Culture and screening were performed.(Peter et.al.,1975)

\section{In Vivo Cultivation of Plasmodium Berghei}

To test the antimalarial activity of the aqueous and alcoholic extracts of each plant, the mouse was infected with, CQ sensitive strains of $P$. berghei (obtained as a kind gift from Dr. S Sharma from Friendicoes, New Delhi) maintained in vivo was used. The parasites were maintained by serial passage of blood from infected mice to the non-infected ones on weekly basis.Donor Plasmodium berghei infected mice (parasitemia of 20\%) were killed by cervical dislocation. Blood was then collected in slightly heparinized syringe from auxillary vesssels. The collected blood was diluted with PBS in such a way that each $0.2 \mathrm{ml}$ contains approximately $1 \times 10^{7}$ infected red cells. Mice were then divided in to ten groups of six each; eight groups of mice received the crude extracts or fractions, while the other two groups were used as positive and negative control (Peters et.al., 1975).

\section{In Vivo Evaluation of The Antimalarial Activity of Natural and Synthetic Drugs In Free and Encapsulated Form}

For an in vivo evaluation of each extract the Peter's 4 day suppressive test against $P$. berghei infection in mice was employed Peters et al 1975 Male Swiss albino mice weighing 27-32g were infected with $P$. berghei and randomly divided into eight groups of five mice per cage: eight test groups(extracts,drugs and drug delivery systems) (G8-CQ a standard drugs as a positive control and G1-vehicles, $\mathrm{dH}_{2} 0$ or $3 \%$ DMSO, as a negative control). Each mouse received standard inoculums of about $10^{6}$ $10^{7}$ parasite infected red cells per gram body weight through the intraperitoneal route in a PBS of $0.2 \mathrm{ml}$ on D0 to produce a steadily rising infection in mice. The stock of aqueous extracts were made dissolved in distilled water $\left(\mathrm{dH}_{2} \mathrm{O}\right)$ and ethanolic extracts 
were dissolved in less than 3\% DMSO according to the weight of mice in each group. The extracts were given via intragastric route by using gavage in six different doses $\left(100,200,400,600,800\right.$ and $\left.1000 \mathrm{mg} \mathrm{kg}^{-1} \mathrm{day}^{-1}\right)$, while the positive control mice received chloroquine (CQ) at $25 \mathrm{mg}^{-1} \mathrm{~kg}^{-1}$ day and the negative control received vehicles with in a volume of $0.2 \mathrm{ml}$. Each treatment was administered as a single dose per day and was started after 3 hours of infection on day zero continued daily for four days. Peters et al 1975 On the fourth day drops of blood sample was collected from the tail snip of each mouse. Thin smears were prepared and stained with $10 \%$ Giemsa solution. Then, each stained slide was examined under microscope with an oil immersion magnification (100x) power to evaluate the percentage of parasitaemia and suppression of each extract with respect to the control groups.LD50 was determined (Dikasso et.al.,2006)

\section{Results and Discussion}

P.falciparum culture was maintained in RPMI 1640 medium supplemented with 5\% fresh human serum of blood group $\mathrm{O}^{+}$(with serum of blood $\mathrm{AB}^{+}$group P.falciparum growth rate was slow), $3 \%$ sodium bicarbonate and $25 \mathrm{mM}$ HEPES buffer in $\mathrm{CO}_{2}$ rich conditions. Plasmodium falciparium culture was maintained with regular change of medium (every 16-18 hrs) to achieve maximum growth. Cultures were synchronized with sorbitol before starting the screening assay (to get uniform cell growth).

All extracts which were to be screened for the anti plasmodium activity in vitro model were resuspended in DMSO (1 $\mathrm{mg} \mathrm{ml}^{-1}$ stock), dilutions was prepared from the stock (under aseptic conditions) all doses were chosen based on a pre dose finding test for extracts from the literature values for plant. $\mathrm{LD}_{50}$ obtained for each extract are given in Table 1.

Table 1: $\mathrm{LD}_{50}$ of Plant Extracts In Vitro Screening

\begin{tabular}{|c|c|c|}
\hline S.No & Plant Extract & LD $_{50}$ value $\left(\mu \mathrm{g} \mathrm{ml}^{-1}\right)$ \\
\hline 1 & Azadirachta indica (alcoholic extract) & 6.52 \\
\hline 2 & Azadirachta indica (Aqueous extract) & 350 \\
\hline 3 & Cinchona officinalis (alcoholic extract) & 8.76 \\
\hline 4 & Cinchona officinalis (Aqueous extract) & 375 \\
\hline 5 & Artemisia anпua (alcoholic extract) & 4.2 \\
\hline
\end{tabular}

Similarly synthetic drugs were screened for activity against P.falciparum culture maintained in laboratory. Culture was synchronized with sorbitol treatment before starting the screening protocol. $\mathrm{LD}_{50}$ of drugs are given in Table 2 calculated with probit graph . Within each experiment, standard deviations are typically less than $10 \%$ of the mean. Differences in parasite stages of development which can lead to up to two-fold shifts in the $\mathrm{LD}_{50}$ values between experiments; however, these differences rarely affect the overall relationships between the parasite lines in terms of their differences in drug response. From preliminary studies, indicated that aqueous extracts of $A$. indica and aqueous extract of $C$. officinalis and A.annua displayed no activity or 
very less, against the $P$. berghei .Although some positive results were seen when used with drug delivery systems. Indicate that when extracts were introduced in system with encapsulating polymers, there is a supportive change in pharmacodynamic nature of compounds, or encapsulating polymer might protect active constituents form detoreation due to interference from host immune response,one more factor may contribute to effective results is that delivery sytems can target compound to the site of infection,or uses channels which are used by Plasmodium for its growth and metabolism.

Table 2: $\mathrm{LD}_{50}$ of synthetic drug in vitro screening:

\begin{tabular}{lll}
\hline S.No & Drug name & LD $_{\mathbf{5 0}}$ Value in $\mathbf{~ n ~}^{\mathbf{1}} \mathbf{~}^{-1}$ \\
\hline 1 & Chloroquine & 57.34 \\
2 & Artesunate & 32.56 \\
3 & Hafloquine & 47.62 \\
\hline
\end{tabular}

The hand shaking, film method was used due to its simplicity, reproducibility and high efficiency of aqueous phase encapsulation. Sonication and extrusion were used for homogenization of vesicle size distribution of MLV liposomes obtained by the film method. Bath sonication reduced the size of MLVs. Mean particle size-results of empty and drug/extract loaded liposomes with or without cholesterol for liquid and gel type with Phospholipid: charge inducer: cholesterol in the composition of $(10: 1$ : 4 ) and $(7: 1: 2)$, were tried for the preparation of bilayers(Formulations are discussed in Table 3) in order to get higher encapsulation of the drug. Since bilayer composition is one of the effecting factors on encapsulated drug amount (Crommelin 1984), (10: 1: 4) was found to encapsulate higher drug than (7:1:2). For (10:1:4), (soya lecithine 10, cholesterylhemisuccinate 1,cholesterol 4), for (10:1), (soya lecithine 10, cholesterylhemisuccinate,1), for (7:1:2), (soyalecithine 7 cholesterylhemisuccinate 1,cholesterol2),for(7:1), (soya lecithine 7, cholesterylhemisuccinate,1). For homogenization of the vesicles, sonication were used.

Table 3: Mean particle size results of empty and loaded liposomes in present study, with or without cholesterol, liquid and gel state in molar ratios of (10:1:4), (10: 1$),(7: 1: 2)$ and $(7: 1)$

\begin{tabular}{llll}
\hline S.No & Formulation & Molar ratio & Mean particle size $\boldsymbol{\mu m} \pm$ SD \\
\hline 1 & L1(empty) & $10: 1: 4$ & $3.8 \pm 0.02$ \\
2 & L2(loaded with extract) & $10: 1: 4$ & $3.6 \pm 0.03$ \\
3 & L3(loaded with drug) & $10: 1: 4$ & $3.9 \pm 0.07$ \\
4 & L4(with out cholesterol) & $10: 1$ & $3.5 \pm 0.04$ \\
5 & L5(empty) & $7: 1: 2$ & $3.7 \pm 0.06$ \\
6 & L6(loaded with extract) & $7: 2: 1$ & $3.6 \pm 0.03$ \\
7 & L7(loaded with drug) & $7: 2: 1$ & $3.2 \pm 0.07$ \\
8 & L8(with out cholesterol) & $7: 2$ & $3.7 \pm 0.05$ \\
\hline
\end{tabular}


Liposomes suspension was digested with minimum amount of ethanolic solution. Then digested homogenates were centrifuged at $12000 \mathrm{rpm}$ for $30 \mathrm{~min}$ and supernatant was analysed for drug entrapment.PBS of pH 7.4 was used as suspension media. Entrapped drug (Table.4) and extracts in this study were measured at their respective $\lambda$ maximum.

Table 4: Results of encapsulation efficiency of extracts and synthetic drugs Liposomes

\begin{tabular}{lll}
\hline S.No & Drug/Extract & $\begin{array}{l}\text { Encapsulation } \\
\text { efficiency \% }\end{array}$ \\
\hline 1 & Azadirachta indica (aqueous extract) & 88.74 \\
2 & Azadirachta indica (alcoholic extract) & 89.83 \\
3 & Cinchona officinalis (alcoholic extract) & 89.64 \\
4 & Artemisia annua (alcoholic extract) & 95.74 \\
5 & Chloroquine & 99.75 \\
6 & Mefloquine & 98.45 \\
7 & Artesunate & 98.36 \\
\hline
\end{tabular}

Table 5: Results for cumulative in vitro release of synthetic drugs encapsulated in liposomes

\begin{tabular}{lllll}
\hline S.No & Time (h) & $\begin{array}{l}\text { Cumulative } \\
\text { drug \% release } \\
\text { Chloroquine }\end{array}$ & $\begin{array}{l}\text { Cumulative } \\
\text { drug \% release } \\
\text { Mafloquine }\end{array}$ & $\begin{array}{l}\text { Cumulative } \\
\text { drug \% release } \\
\text { Artesunate }\end{array}$ \\
\hline 1 & 1 & 29.85 & 34.94 & 37.96 \\
2 & 2 & 37.94 & 42.94 & 45.93 \\
3 & 3 & 48.94 & 49.84 & 48.74 \\
4 & 4 & 52.72 & 55.83 & 53.94 \\
5 & 5 & 59.95 & 62.93 & 59.28 \\
6 & 6 & 65.73 & 69.53 & 63.75 \\
7 & 7 & 72.84 & 73.84 & 69.73 \\
8 & 8 & 79.84 & 78.54 & 75.79 \\
9 & 9 & 86.93 & 83.63 & 89.72 \\
10 & 10 & 92.84 & 87.64 & 95.74 \\
\hline
\end{tabular}

In vitro release of extract out of liposomes was measured (Graph: 1)spectrophotometrically by incubation of liposomes in $10 \mathrm{mM}$ Tris buffer ( $\mathrm{pH}:$ 7.4) at $37^{\circ} \mathrm{C}$ in dylising bags with mild shaking $(35 \mathrm{rpm})$ water bath. Samples were withdrawn at time intervals of $1,2,4,6,8,10 \mathrm{~h}$, periodically. In vitro release rate results (Fig: 1) were evaluated kinetically (Zero, first-order and Higuchi models).Similarly Cumulative percentage of in vitro release of synthetic drugs encapsulated in liposomes was calculated results (Table 5) indicated 
Table 6: Cumulative percentage of in vitro release of extract encapsulated in liposomes

\begin{tabular}{llllll}
\hline S.No & $\begin{array}{l}\text { Time } \\
\text { (h) }\end{array}$ & $\begin{array}{l}\text { Cumulative } \\
\text { \% \% release } \\
\text { Azadirachta } \\
\text { indica } \\
\text { aqueous }\end{array}$ & $\begin{array}{l}\text { Cumulative } \\
\text { \% release } \\
\text { Azadirachta } \\
\text { indica } \\
\text { alcoholic }\end{array}$ & $\begin{array}{l}\text { Cumulative } \\
\text { \% release } \\
\text { Cinchona } \\
\text { officinalis }\end{array}$ & $\begin{array}{l}\text { Cumulative } \\
\text { \% release } \\
\text { Artemisia } \\
\text { extract }\end{array}$ \\
\hline 1 & 1 & 35.85 & 39.42 & 38.56 & 32.73 \\
2 & 2 & 43.64 & 46.82 & 42.74 & 39.53 \\
3 & 3 & 49.74 & 52.52 & 56.62 & 49.43 \\
4 & 4 & 53.64 & 59.82 & 63.94 & 59.63 \\
5 & 5 & 59.74 & 64.42 & 69.66 & 65.74 \\
6 & 6 & 64.72 & 69.82 & 74.74 & 70.32 \\
7 & 7 & 72.73 & 75.83 & 83.68 & 75.73 \\
8 & 8 & 79.72 & 81.94 & 87.64 & 82.43 \\
9 & 9 & 86.76 & 88.82 & 90.54 & 88.84 \\
10 & 10 & 94.73 & 92.06 & 94.52 & 92.65 \\
\hline
\end{tabular}

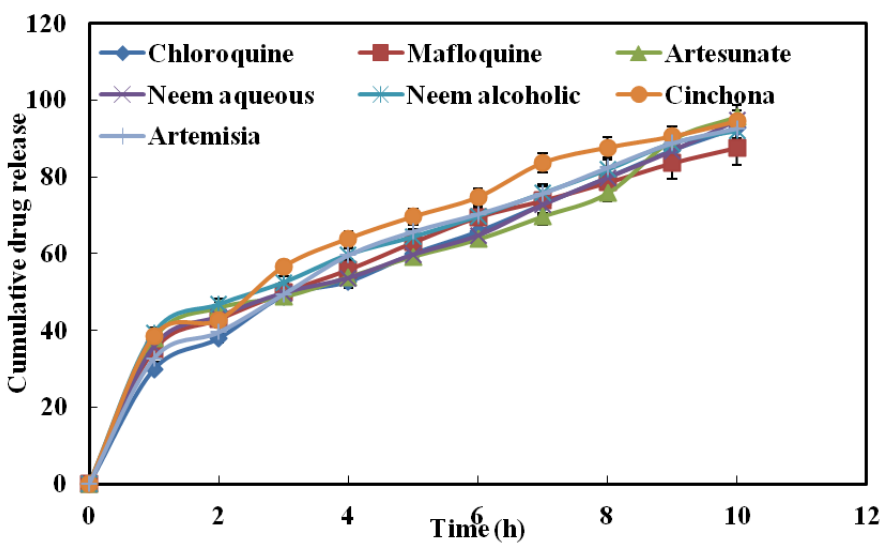

Figure 1: Cumulative drug release of liposomes in vitro model

$\lambda$ max of Azadirachta indica extract : $577 \mathrm{~nm}$,for Cinchona officinalis extract : $250 \mathrm{~nm}$, for Artemisia annua extract: $365 \mathrm{~nm}$, for Chloroquine: $343 \mathrm{~nm}$, for Mefloquine:283nm,for Artesunate : $287 \mathrm{~nm}$.

In vitro drug release for liposomes in present study suggest that,there is controlled release pattern of all the molecules studied,which contribute to affecient release of drug to the target side reducing the requirement of drug for parasitic control.Results are satisfactory for liposomes as effective and novel drug delivery system.

Anti-malarial drugs have been encapsulated in liposomes with the objective of protection of the drug from the degradation and the sustained release of the drug along with minimization of its adverse effects. Gabriels et al.,(2003) have formulated 
Artesunate liposomes to reduce its dosing frequency and they found 30\% release in 24 $\mathrm{h}$, in the in vitro release test using the dialysis bag technique. Encapsulation of $\beta$ artemether in liposomes for the treatment of recrudescent malaria was reported by Chimanuka et al., (2002). The egg phosphatidylcholine cholesterol liposomes prepared by the group with $100 \%$ entrapment efficiency was demonstrated to successfully circumvent the recrudescent parasitemia in Plasmodium chabaudiinfected mice. They had reported that additive effect of phosphatidylcholine in inducing immune response against the parasite infection.

Oral administration of arteether encapsulated in dipalmitoyl- phosphatidylcholine, dibehynoylphophatidylcholine, cholesterol liposomes formulated by Bayomi et al., (1998) remained for a longer time in gastrointestinal tract and showed higher bioavailability, higher Cmax and shorter Tmax as well as higher value of AUC when compared with its aqueous suspension in New Zealand rabbits. These liposomes could also be administered intravenously and showed longer elimination half-life. Chloroquine-encapsulated liposomes with or without surface modification have been studied by different groups. Owais et al., (1995) have tagged antibody against infected erythrocytes on the chloroquine liposomes and tested their efficacy in chloroquine-resistant Plasmodium berghei infection in mice. The chloroquine delivered in these liposomes intravenously at a dosage of $5 \mathrm{mg} \mathrm{kg}^{-1}$ of body weight per day on days 4 and 6 post-infection completely cured the animals (75\% to $90 \%)$ of chloroquine-resistant $P$. berghei infections. These results indicate that selective homing of chloroquine to malaria-infected erythrocytes may help to cure the chloroquine resistant malarial infections with reduced dose. Similar results were obtained by Crommelin et al., (1991).

\section{Conclusion}

Present study confirms the effectiveness of liposomes as drug delivery system for selected antimalarial compounds.Drug delivery system reduces drug dosage requirement due to control drug releasing property. All the three plants selected for the anti plasmodium activity in present study are known for their medicinal property againt various disease including antimalarial.In vitro model proves their activity against P.falciparum.when tested against the P.berghei results were not satisfactory for extracts of Azadirachta indica,but its efficiency increases when encapsulated with drug delivery systems used in present study.Drug delivery systems proves their efficiency for control release of drug to the system,contributing to less drug requirement for efficient effect,and low or no toxicity due to high dose of drug.

\section{References}

[1] Maurer N, Fenske D.B., Cullis P.R., (2001) Developments in liposomaldrug delivery systems. Expert Opinion on Biological Therapy 1:923-947.

[2] Trease G E and Evans W C. Pharmacology. (1978) 11th Ed. Bailliere Tindall Ltd, London pp 60-75 
[3] Kokate C K, Purohit A P, Gokhale S B. (2009) Pharmacognosy. Nirali Prakashan. 6; pp6-17.

[4] Trager W, Jenson J B.(1997) Continous culture of Plasmodium falciparum, its impact on malarial research. International Journal for Parasitology ;27(9): 989-1006.

[5] Peters W, Portus J H, Robinson B L.(1975). The chemotherapy of rodent malaria, XXII. The value of drug-resistant strains of Plasmodium berghei in screening for blood schizontocidal activity. Annals of Tropical Medicine and Parasitology; 69: 155-171.

[6] Martinelli A, Rodrigues L A, Cravo P.(2008). Plasmodium chabaudi: efficacy of artemisinin + curcumin combination treatment on a clone selected for artemisinin resistance in mice. Experimental Parasitology; 119 (2):304-307.

[7] Crommelin D.J.A., Eling W.M.C., Steerenberg P.A., Nässander U.K., Storm G., DeJong W.H.,. Van Hoesel Q.G.C.M, Zuimeda J., (1991). Liposomes and immunoliposomes for controlled release or site specific delivery of antiparasitic drugs and cystostatics, Journal of Controlled Release 16: 147154.

[8] Dikasso Dawit, Makonnen Eyasu M, Debella Asfaw, Urga Kelbesa, Wallelegn Mekonen, Daniel Melaku,Moges kassa and Mulugeta Guta .(2006). Antimalarial activity of Withania somnifera L.Dunal extracts in mice. Ethiopian Journal of Health Development ; 44: 279.

[9] Vyas S P and Khar R K (2002).Targeted and controlled drug delivery, CBS publishers and Distributors pp331-386.

[10] Trotta M, Debernardi F, Caputo O.(2003) Preparation of solid lipid nanoparticles by a solvent emulsification-diffusion technique. International Journal of Pharmaceutics. 257: 153-160.

[11] Maurer N, Fenske D B, Cullis P R (2001). Developments in liposomaldrug delivery systems. Expert Opinion on Biological Therapy. 2001; 1: 923-947.

[12] Vemuri S, Yu CD, Wangsatorntanakun V, Roosdorp N. (1990).Large-scale production of liposome by a microfluidizer. Drug Dev Ind Pharm. ; 16(15):2243-2256.

[13] Owais M.,Varshney G.C.,Choudhury A.,Chandra S., Gupta C.M.,(1995).Chloroquine encapsulated in malaria-infected erythrocytespecific antibody-bearing liposomes effectively controls chloroquine-resistant Plasmodium berghei infections in mice, Antimicrobial Agents and Chemotherapy. 39: 180-184

[14] Chimanuka B, Gabriëls M, Detaevernier M.R, Plaizier J.A,(2002). Vercammen,Preparation of $\beta$-artemether Liposomes, their HPLC-UV evaluation and relevance for clearing recrudescent parasitemia in Plasmodium chabaudi malaria-infected mice, Journal of Pharmaceutical and Biomedical Analysis. 28: 13-22.

[15] Gabriels M., Plaizier-Vercammen J., (2003). Physical and chemical evaluation of Liposomes, containing Artesunate, Journal of Pharmaceutical and Biomedical Analysis. 31:655-667.

[16] Bayomi M.A.,Al-Angary A.A., Al-Mehsal M.A., Al-Dardiri M.M., (1998). Invivo evaluation of arteether Liposomes, International Journal of Pharmaceutics. 175: 1-7. 